\title{
UCRL-JRNL-202507
}

LAWRENCE LIVERMORE NATIONAL LABORATORY

\section{Effects of the Electron Energy Distribution Function on Modeled X-ray Spectra}

S. B. Hansen and A. S. Shlyaptseva

$3 / 22 / 04$

Submitted to Physical Review E 
This document was prepared as an account of work sponsored by an agency of the United States Government. Neither the United States Government nor the University of California nor any of their employees, makes any warranty, express or implied, or assumes any legal liability or responsibility for the accuracy, completeness, or usefulness of any information, apparatus, product, or process disclosed, or represents that its use would not infringe privately owned rights. Reference herein to any specific commercial product, process, or service by trade name, trademark, manufacturer, or otherwise, does not necessarily constitute or imply its endorsement, recommendation, or favoring by the United States Government or the University of California. The views and opinions of authors expressed herein do not necessarily state or reflect those of the United States Government or the University of California, and shall not be used for advertising or product endorsement purposes.

This work was performed under the auspices of the U.S. Department of Energy by University of California, Lawrence Livermore National Laboratory under Contract W-7405-Eng-48. 


\title{
Effects of the electron energy distribution function on modeled x-ray spectra
}

\author{
S. B. Hansen \\ Physics Department/220, University of Nevada, Reno, Nevada, 89557 and \\ Lawrence Livermore National Laboratory, PO Box 808, L-260, Livermore, CA 94551
}

\begin{abstract}
A. S. Shlyaptseva
Physics Department/220, University of Nevada, Reno, Nevada, 89557
\end{abstract}

This paper presents the results of a broad investigation into the effects of the electron energy distribution function on the predictions of non-LTE collisional-radiative atomic kinetics models. The effects of non-Maxwellian and suprathermal ("hot") electron distributions on collisional rates (including three-body recombination) are studied. It is shown that most collisional rates are fairly insensitive to the functional form and characteristic energy of the electron distribution function as long as the characteristic energy is larger than the threshold energy for the collisional process. Collisional excitation and ionization rates, however, are highly sensitive to the fraction of hot electrons. This permits the development of robust spectroscopic diagnostics that can be used to characterize the electron density, bulk electron temperature, and hot electron fraction of plasmas with non-equilibrium electron distribution functions (EDFs). Hot electrons are shown to increase and spread out plasma charge state distributions, amplify the intensities of emission lines fed by direct collisional excitation and radiative cascades, 
and alter the structure of satellite features in both K- and L-shell spectra. The characteristic energy, functional form, and spatial properties of hot electron distributions in plasmas are open to characterization through their effects on high-energy continuum and line emission and on the polarization of spectral lines.

32.30.Rj, 32.70.Fw, 52.20.Fs, 52.25.-b

\section{INTRODUCTION}

Non-Maxwellian and suprathermal (or "hot") electrons are of a topic of continuing interest to the plasma physics, fusion, and astrophysical communities because they can play an important role in the formation, evolution, and radiative properties of a wide variety of plasma sources. Non-Maxwellian electron energy distributions have been predicted or detected in diverse laboratory sources including tokamaks, ${ }^{12}$ laser plasmas, 345 and pulsed-power plasmas, ${ }^{5678}$ as well as in astrophysical sources such as solar flares 91011 and active galactic nuclei, ${ }^{12}$ where they are generated by strong electric fields or resonant laser-plasma interactions. In addition, collisional x-ray sources that measure basic atomic processes, such as electron beam ion traps (see, for example, ${ }^{13}$ ) are typically driven by electron beams, which are fundamentally non-Maxwellian.

Collisional-radiative atomic models that include the effects of non-Maxwellian and suprathermal electron energy distributions are therefore of significant interest, both in benchmarking atomic physics data and as spectroscopic tools that can determine the 
presence and characteristics of the electrons distribution function (EDF) in plasmas from non-invasive spectroscopic measurements.

Understanding the role of hot electrons in plasmas is particularly important because of their influence on plasma dynamics, radiation production, and energy balances. A major historical driver in the study of plasmas with hot electrons is the issue of preheat in laserdriven inertial confinement fusion schemes. Intense lasers interacting with dense targets can deposit significant energy in hot electrons through resonant absorption; ${ }^{14}{ }^{15}$ such electrons can lead to significant energy losses and have deleterious effects on plasma stability and control (see, for example, ${ }^{16}$ ). In other cases, the effects of hot electrons are desirable, such as when they enhance the output of high-energy radiation from relatively low-energy plasma sources. ${ }^{5171819}$ Whether hot electrons are beneficial or harmful, detecting and characterizing them in laboratory plasmas is an important step towards controlling their effects.

The effects of hot electrons on modeled K-shell line spectra have been extensively studied using two-temperature electron distribution functions. ${ }^{20}{ }^{21}$ Hot electrons have been shown to affect K-shell spectra from Al X pinches, ${ }^{17}$ Ar plasma focus devices, ${ }^{22}$ and plasmas produced by laser irradiation of solid Mg, ${ }^{23}$ Ar gas clusters, ${ }^{18} 242526$ and Ti-doped hohlraums. ${ }^{27}$ More recently, L-shell spectroscopic diagnostics for hot electrons have been developed for $\mathrm{Kr},{ }^{28} \mathrm{Cu},{ }^{29}{ }^{30}$ and $\mathrm{Zn}{ }^{30}$ laser plasmas and for Mo X pinch plasmas. ${ }^{19}$ Both the K- and L-shell studies have determined that hot electrons increase 
the average charge state balance of the plasmas and amplify the intensities of emission lines formed through direct collisional excitation.

These studies of hot electrons have so far been tailored to particular experiments, and their conclusions have been limited to fixed forms of the energy distribution used to describe the hot electrons. Gaussian electron energy distributions have been used to describe hot electrons generated by intense laser pulses on gas cluster targets, 1824252628 hot electrons in plasmas formed by laser irradiation of solid targets have been modeled with both Gaussian ${ }^{23}$ and Maxwellian 272930 distribution functions, and hot electrons in pulsed-power plasmas have been modeled using Gaussian ${ }^{17} 22$ and power-law ${ }^{19}$ distributions.

This work is to our knowledge the first study of the influence of a wide range of electron distribution functions on both $\mathrm{K}$ - and L-shell spectra. It in part reprises and confirms the previous studies and also extends and generalizes their results. Section II introduces the K- and L-shell collisional-radiative models and the three functional forms of the electron distribution on which the present analysis is based. In Section III, the variations of several broad categories of collisional rate coefficients with the characteristic energy and functional form of the electron distribution function are presented. In section IV, the effects of hot electrons on plasma charge state balances and the sensitivity of plasmas composed of elements with $10<Z<50$ to small fractions of hot electrons are investigated. Section V presents the effects of hot electrons on K- and L-shell line 
emission, and the effects of hot electrons on continuum and high-energy inner-shell line emission are investigated in Section VI. A summary is given in section VII.

\section{DESCRIPTION OF COLLISIONAL-RADIATIVE MODELS AND ELECTRON DISTRIBUTION FUNCTIONS}

Non-LTE collisional-radiative atomic kinetics models are widely used to diagnose plasma conditions by matching measured to modeled x-ray spectra. The modeled spectra are dependent on modeled charge state balances and energy level populations, which are in turn dependent on the electron distribution function through its effect on collisional rates.

In this work, the effects of varying the electron distribution function on modeled charge state balances and line and continuum x-ray spectra are investigated using a model of Kshell Ti and models of L-shell $\mathrm{Kr}$ and Mo. The L-shell models include detailed fine structure levels for O-like through Mg-like ions and ground states from the bare ions to the neutral atoms. The data sources, level structure, and coupling details of the L-shell models have been described in detail elsewhere. ${ }^{19} 2831$ The K-shell Ti model is based on

atomic data calculated using the new FAC atomic structure code. ${ }^{32}$ It includes the ground states of all ions, singly excited levels up to $n=5$ for $\mathrm{H}$ - and H-like ions, $n=4$ for Li-like ions, and $n=3$ for Be-through Ne-like ions, and doubly excited levels up to $n=3$ for He- 
, Li-, and Ne-like ions and up to $n=2$ for Be- through F-like ions. All levels are coupled within each ion by radiative decay and collisional excitation and de-excitation and are coupled between neighboring ions with collisional ionization, Auger decay, and their reverse rates as well as by radiative recombination.

Collisional rates are calculated in the models using one of three basic forms of the electron energy distribution function $F_{X}\left(\varepsilon ; \varepsilon_{0}\right)$ :

$$
\begin{array}{lc}
\text { Maxwellian: } & F_{M}\left(\varepsilon ; T_{e}\right)=2 \sqrt{\frac{\varepsilon}{\pi T_{e}^{3}}} \exp \left[-\varepsilon / T_{e}\right] \\
\text { Gaussian: } & F_{G}\left(\varepsilon ; \varepsilon_{0}\right)=\frac{1}{w \sqrt{\pi}}\left(\frac{2}{1-\operatorname{erf}\left(-\varepsilon_{0} / w\right)}\right) \exp \left[-\left(\frac{\left(\varepsilon-\varepsilon_{0}\right)}{w}\right)^{2}\right] \\
\text { Power-law: } & F_{P}\left(\varepsilon ; \varepsilon_{0}\right)=\left(\frac{\gamma-1}{\varepsilon_{0}^{1-\gamma}}\right) \varepsilon^{-\gamma} ; \varepsilon \geq \varepsilon_{0}
\end{array}
$$

The distributions in (1) - (3) are given in terms of the electron energy $\varepsilon$ and characteristic distribution energy $\varepsilon_{0}$ in their normalized form $\left(\int F_{X}\left(\varepsilon ; \varepsilon_{0}\right) d \varepsilon=1\right)$. The characteristic energy of the Maxwellian (thermal) distribution (1) is equivalent to an electron temperature $T_{e}$. The Gaussian distribution (2), which can represent electrons that are excited by resonant laser-plasma interactions, is centered at a characteristic energy $\varepsilon_{0}$ and has a half-width at half-maximum of $w \sqrt{\ln 2}$. (The term in square brackets in (2) containing the error function can be neglected when $\varepsilon_{0} \gg>w$ and is included here only so that cases with $w \sim \varepsilon_{0}$ can be treated rigorously.) The power-law distribution (3), which is commonly used in astrophysical models, becomes nonzero at its characteristic electron energy $\varepsilon_{0}$ and decays more or less rapidly with energy according to the value of $\gamma$. The 
discontinuity in the power-law distribution at $\varepsilon_{0}$ may be unphysical, however, for most two-temperature plasmas $\varepsilon_{0}$ can be chosen so that the hot electron power-law distribution merges smoothly with a cool Maxwellian distribution.

Variations of the electron distributions given by equations (1) - (3) are shown in Fig. 1: three Maxwellian distributions with $T_{e}=0.1,1.0$, and $10 \mathrm{keV}$, two Gaussian distributions with $\varepsilon_{0}=10 \mathrm{keV}$ and widths $w=0.1$ and $1.0 \mathrm{keV}$, and two power-law distributions with $\varepsilon_{0}=10 \mathrm{keV}$ and decay constants $\gamma=2$ and 5 . An extremely wide range of electron energy distributions can be modeled by combining these three functional forms. The present study is restricted to electron distributions composed of either a single functional form of the distributions given in (1) - (3) or a two-temperature distribution with both a bulk Maxwellian component $F_{M}\left(\varepsilon ; T_{e}\right)$ and a fraction $f$ of electrons in a second distribution $F_{X}\left(\varepsilon ; \varepsilon_{0}\right)$ :

$$
F(\varepsilon)=(1-f) F_{M}\left(\varepsilon ; T_{e}\right)+f F_{X}\left(\varepsilon ; \varepsilon_{0}\right)
$$

Once the electron energy distribution function is specified, collisional rates are calculated as described in the following section and used along with spontaneous radiative and Auger decay rates in the collisional-radiative models to obtain steady-state energy level populations. These energy level populations are used to determine the charge state balance of the modeled plasma and to construct optically thin line and continuum emission spectra. 


\section{EFFECTS OF EDF ON COLLISIONAL RATES}

The rates of collisional process in a plasma are given by the product of the plasma electron density $n_{e}$ and the rate coefficient $\langle v \sigma\rangle$ of the particular process. The rate coefficient is determined by integrating an energy-dependent collision cross section $\sigma(\varepsilon)$ over the electron energy distribution function:

$$
\langle v \sigma\rangle=\int_{\Delta E}^{\infty} v \sigma(\varepsilon) F(\varepsilon) d \varepsilon \quad\left[\mathrm{cm}^{3} \mathrm{~s}^{-1}\right]
$$

In (5), $v$ and $\varepsilon$ are the velocity and energy, respectively, of the incident electron. The lower limit of the integration is the threshold energy of the process for collisional excitation and ionization.

For single-temperature plasmas with Maxwellian electron energy distributions, deexcitation and recombination rates can be obtained directly from collisional excitation and ionization rate coefficients through detailed balance. For plasmas that have electrons in non-Maxwellian distributions, the cross sections of these reverse rates must be integrated over the entire electron energy distribution. Collisional de-excitation cross sections $\sigma_{k j}^{d e x}$ can be obtained from excitation cross sections $\sigma_{j k}^{e x}$ using the KleinRosseland formula:

$$
g_{k} \varepsilon \sigma_{k j}^{d e x}(\varepsilon)=g_{j}(\varepsilon+\Delta E) \sigma_{j k}^{e x}(\varepsilon+\Delta E)
$$

where $g_{j}$ and $g_{k}$ are the statistical weights of the upper and lower levels, respectively, of the collisional process. Radiative recombination cross sections can be expressed in a similar manner either from photoionization cross sections and the Milne formula or by 
approximations such as the Kramers formula. ${ }^{33}$ Dielectronic recombination is a resonant process whose cross sections can be obtained from Auger decay rates and expressed as delta functions, making the integration in (5) numerically straightforward. Three-body recombination cross sections and rates are less straightforward and are treated in detail in the Appendix.

Figure 2 shows rate coefficients for representative collisional processes integrated over a variety of electron distributions as a function of the characteristic distribution energy $\varepsilon_{0}$ in units scaled to the transition energy $\Delta E$. Rate coefficients typical of collisional ionization and resonant collisional excitation, whose cross sections decay asymptotically as $\ln [\varepsilon] / \varepsilon$, are given in Fig. 2 (a). Non-resonant excitation cross sections tend to decay more quickly with the impact electron energy, with asymptotic dependences on powers of $1 / \varepsilon$, rate coefficients for such processes are given in Fig. 2 (b) and (c). Finally, Fig. 2 (d) shows rate coefficients for collisional de-excitation processes whose cross sections decay as $1 / \varepsilon$ (an asymptotic dependence similar to that of radiative recombination cross sections in the Kramers approximation).

From Fig. 2 (a) - (c), it is plain that collisional excitation and ionization rates are strongly dependent on the functional form and characteristic energy of the electron energy distribution when the characteristic distribution energy $\varepsilon_{0}$ is smaller than the threshold transition energy $\Delta E$. This is understandable because of the wide variations in the number of electrons with sufficient energy to induce the transition among the various distributions. When $\varepsilon_{0}<<\Delta E$, the narrowest distributions (the Gaussian with $w=0.1 \Delta E$ 
and the power-law function with $\gamma=5$ ) include very small numbers of electrons with energies larger than the threshold energy for excitation and give much smaller rate coefficients than the broader distributions. As the characteristic energies increase, all distributions accumulate larger numbers of energetic electrons and the rate coefficients for excitation/ionization processes increase accordingly.

It is important to note that when the characteristic energies of the electron distributions are larger than the threshold energy $\Delta E$, much of the strong dependence of the rate coefficients on the functional forms and characteristic energies of the distributions vanishes. In particular, the ionization and resonant collisional excitation rate coefficients shown in Fig. 2 (a) are only weakly dependent on the characteristic energy and functional form of the electron distribution as long as $\varepsilon_{0} \gg \Delta E$. Only when the cross section decays very rapidly (as in Fig. 2 (c) with $\sigma^{e x}(\varepsilon) \sim 1 / \varepsilon^{3}$ ) do the functional forms and characteristic energies of the distributions have significant impact on the rate coefficients. ${ }^{28}$ Deexcitation and radiative recombination rate coefficients are fairly insensitive to the functional form of the electron energy distribution for all characteristic energies, because those cross sections are integrated over the entire EDF.

The relative insensitivity of collisional ionization, resonant excitation, and radiative recombination rates to the characteristic energy and functional form of the electron distribution when $\varepsilon_{0} \gg \Delta E$ has important consequences for two-temperature collisionalradiative models. Such models typically include hot electron distributions whose characteristic energies are much larger than the largest transition of interest, and the 
insensitivity of hot electron effects to $\varepsilon_{0}$ was noted as early as $1986^{34}$ and has been observed separately for Gaussian ${ }^{1728} 35$ and Maxwellian distributions. ${ }^{29}$

The present analysis implies that even models using different functional forms to describe hot electron distributions should be directly comparable; that the model predictions should be highly dependent on the fraction of hot electrons and only weakly dependent on the functional form and characteristic energy of the hot electron distribution as long as the characteristic energy is larger than (roughly) $Z^{2} R y$, the scaled ionization energy of the H-like ion).

For collisional-radiative models using a two-temperature electron distribution function composed of a cool bulk Maxwellian and a small fraction of hot electrons in an arbitrary energy distribution as in equation (4), the rate coefficient for each collisional process is:

$$
\left[(1-f)\left\langle v^{M} \sigma\right\rangle+f\left\langle v^{X} \sigma\right\rangle\right]
$$

In (7), superscripts on the incident electron velocity indicate the distribution over which the integration is performed (e.g. $v^{M}$ indicates integration over a Maxwellian distribution).

Figure 2 can be used to estimate the effect of a given fraction of hot electrons on a particular collisional rate. For example, the collisional excitation rate for a transition with excitation energy $\Delta E$ in a Maxwellian distribution with $T_{e}=\Delta E / 10$ is more than $10^{4}$ times smaller than the rate from a distribution with $\varepsilon_{0} \geq \Delta E$. Thus, including a fraction $f=$ $10^{-4}$ of hot electrons would roughly double this excitation rate in a plasma with a bulk temperature of $\Delta E / 10$ and a hot electron energy $\varepsilon_{0} \geq \Delta E$. With $f=10^{-3}$, almost $100 \%$ of 
the excitation rate would be due to the hot electrons. For smaller bulk temperatures, even smaller fractions of hot electrons are significant, because cooler Maxwellian distributions have fewer electrons with energies larger than $\Delta E$. Collisional de-excitation and radiative recombination processes are much less sensitive to the presence of hot electrons. As indicated in Fig. 2 (d), energetic electrons are less likely to participate in de-excitation and recombination processes, so the primary effect of a fraction $f$ of hot electrons is to decrease the rates from a bulk Maxwellian distribution by a small factor of about $(1-f)$. Small fractions of hot electrons have similarly small effects on dielectronic and threebody recombination rates.

\section{EFFECTS OF EDF ON CHARGE STATE BALANCES}

Because small fractions of hot electrons have significant impact on collisional excitation and collisional ionization rates and only a small effect on collisional recombination processes, they can significantly increase the charge state balances predicted by collisional-radiative models. It is shown in this section that hot electrons have the additional effect of spreading out modeled charge state balances over a larger number of ions than a typical single-temperature model.

The charge state balances predicted by optically thin collisional-radiative models are in principle dependent on five parameters describing the plasma electrons: $n_{e}, T_{e}, f, \varepsilon_{0}$, and the functional form of the hot electron distribution. Analysis of experimental spectra 
using a model with five parameters informing the collisional rates is significantly more complex than analysis using more typical $n_{e}$ - and $T_{e}$-dependent models. However, the analysis given in the previous section indicates that for characteristic hot electron energies greater than about $Z^{2} R y, \mathrm{CR}$ models should be most sensitive to $n_{e}, T_{e}$, and $f$. This is confirmed in Fig. 3, which shows the effects of fractions of hot electrons on the average charge state $\langle Z>$ of a modeled Ti plasma alongside the effects of changing one of the four other plasma parameters.

In Fig. 3 (a), average charge states calculated using three functional forms of the hot electron distribution with $\varepsilon_{0}=10 \mathrm{keV}$ are shown together: a narrow Gaussian with $w=$ $100 \mathrm{eV}$, a Maxwellian, and a broad power-law function with $\gamma=2$. The values of $<Z>$ obtained with each are almost identical, with the Maxwellian and power-law functions slightly less effective than the Gaussian at ionizing the L-shell ions (which have ionization potential $E_{Z}$ near $1 \mathrm{keV}$ ) and the power-law function slightly more effective than the others at ionizing the K-shell ions (which have $E_{Z}>6 \mathrm{keV}$ ).

Figure 3 (b) shows the effect of changing the characteristic energy of the narrow Gaussian distribution to either $5 \mathrm{keV}$ or $100 \mathrm{keV}$. The overwhelming majority of electrons in the lower-energy ( $5 \mathrm{keV}$ ) Gaussian distribution cannot directly ionize the Kshell ions and lead to smaller average charges than the Gaussian distributions with larger $\varepsilon_{0}$ once the He-like ion with is reached. This underscores the caveat that $\varepsilon_{0}$ should be larger than the largest relevant transition energy. Centering a very narrow distribution at very high energies can also affect the modeled charge state balances: the effect of the100 
$\mathrm{keV}$ Gaussian electrons on the L-shell ionization stages is mitigated by the decay of Lshell collision cross sections at high energies. However, even in the extreme case of a very narrow hot electron distribution, the effects of varying $\varepsilon_{0}$ are small compared to the effect of varying the fraction of hot electrons $f$.

Fig. 3 (c) shows how changing the electron density impacts the effects of hot electrons. For $n_{e}=10^{18}$ and $10^{20} \mathrm{~cm}^{-3}$, the effects of a given $f$ are almost indistinguishable. However, for $n_{e}=10^{22}$ and higher electron densities, increased collisional rates between closely spaced levels can drive population into high-energy levels even at small bulk temperatures (a process referred to as ladder ionization). As collisional processes begin to dominate the kinetics on the approach to LTE, the amplification of direct high-energy collisional processes caused by hot electrons becomes less important. This mitigation of hot electron effects at high electron densities has been noted previously. ${ }^{29} 36$

Finally, Fig. 3 (d) shows how varying the bulk electron temperature from $100 \mathrm{eV}$ to 500 $\mathrm{eV}$ changes the effects of hot electrons in a $10 \mathrm{keV}$ Gaussian distribution. As the temperature increases, the number of energetic electrons in the bulk Maxwellian distribution also increases, requiring larger fractions of hot electrons to generate an observable effect. This was also noted in ${ }^{18}$.

The fact that collisional rates are less sensitive to small fractions of hot electrons at larger bulk electron temperatures because of increasing competition from the bulk electrons can be used to generate a simple estimate of the sensitivity of K-and L-shell ions to hot 
electron fractions. The reported sensitivity of CR models to hot electrons varies widely: K-shell Mg spectra have been shown to be sensitive to fractions of hot electrons as small as $10^{-9},{ }^{23}$ while L-shell Mo spectra are sensitive only to hot electron fractions near $10^{-2}$. ${ }^{19}$ This variation in sensitivity is due not to differences in the energies or functional forms of the hot electron distributions used in the different CR models (which have been shown in Section III to be relatively unimportant) but rather to differences in the bulk electron temperature required to reach the charge states of interest for different elements.

In order for a fraction of hot electrons to affect modeled line spectra, it must be on the order of the fraction of energetic electrons (electrons with $\varepsilon>\Delta E$ ) already present in the bulk Maxwellian. Since elements with larger atomic numbers require larger bulk temperatures to reach a given charge state, and the fraction of energetic electrons scales with the electron temperature, elements with larger atomic numbers will only be sensitive to relatively large fractions of hot electrons. If $T_{0}$ is the bulk electron temperature at which a given element populates the He-like (or Ne-like) charge state, then the sensitivity limit of K-shell (or L-shell) ions is roughly equal to the fraction of Maxwellian electrons in the bulk distribution at $T_{0}$ that have energies greater than the $Z$-scaled ionization potentials of the He-like (or Ne-like) charge state. Figure 4 shows estimated values for the sensitivity limits $\left(f_{\min }\right)$ of L-shell and K-shell spectra for elements with atomic numbers $10<Z<50$ obtained in this way using $T_{0}$ calculated in the coronal approximation. 
The data points given in Fig. 4 are the reported sensitivity limits of various K- and Lshell CR models from many of the references listed in the introduction. Their generally good agreement with the sensitivity estimates determined in the manner detailed above supports the assertion that $\mathrm{CR}$ model predictions are only weakly sensitive to the functional forms and characteristic energies of hot electron distributions, which vary widely among the listed models (as detailed in the introduction). The given sensitivity estimates may also be useful in designing experiments for which hot electron diagnostics are desired and complement the analysis given in, ${ }^{21}$ which recommends that for a given experimental $T_{e}$, the plasma material should be selected to have an atomic number of approximately $0.65 \sqrt{T_{e}(\mathrm{eV})}$ in order for K-shell spectra to be sensitive to hot electron fractions on the order of $1 \%$. A similar selection criterion for L-shell spectra requires $Z \sim$ $1.3 \sqrt{T_{e}(\mathrm{eV})}$

In addition to increasing the average charge states of plasma ions, hot electrons spread out the distribution over more charge states. This effect is illustrated in Fig. 5, which shows the fractional populations of Ti charge states for six EDFs chosen to give average charge states $<Z>$ near 14 (O-like Ti), 17 (B-like Ti), and 20 (He-like Ti). For each $<Z>$, the single-temperature Maxwellian has a smaller number of charge states with significant population than the two-temperature EDF with $T_{e}=100 \mathrm{eV}$ and some fraction of hot electrons at $10 \mathrm{keV}$. That hot electrons can significantly increase populations of $\mathrm{H}$-like $\mathrm{Ti}$ without a great reduction in C- through Be-like Ti was noted in, ${ }^{21}$ and this effect has been used to diagnose hot electron fractions in $1 \mathrm{MA} \mathrm{X}$ pinch plasmas using L-shell Mo spectra ${ }^{19}$ and in laser hohlraums using K-shell Ti spectra. ${ }^{27}$ Finally, hot electrons can 
influence the evolution of plasma charge states and radiation through their impact on excitation and ionization rates. Ionization time scales that would require very high electron densities in a single-temperature plasma may be possible in lower-density plasmas if hot electrons are present. ${ }^{28}$

\section{EFFECTS OF EDF ON K- AND L-SHELL LINE EMISSION}

The effects of hot electrons on the plasma charge state distribution discussed above are manifested in the modeled plasma spectra: hot electrons increase the emission from higher charge states and spread out the number of charge states from which significant emission is seen. In addition, hot electrons can affect the line emission from within particular charge states. Such effects can be used to distinguish the effects of hot

electrons from those of spatial gradients, ${ }^{21}$ opacity, ${ }^{28} 2930$ or other plasma phenomena.

Hot electrons have three primary effects on line emission from a single charge state. First, they can significantly enhance the populations of excited levels and thereby increase the intensity of all line emission, particularly of high-energy lines. Second, they can change the shape of satellite spectra by amplifying collisionally excited inner-shell satellite lines relative to satellite lines populated by dielectronic recombination. Finally, by increasing the total population in excited levels, hot electrons can amplify the intensities of lines fed by radiative cascades, especially in closed-shell ions with relatively few dipole decay channels to the ground state. 
All of the mentioned effects are illustrated in Fig. 6, which shows K-shell Ti spectra at conditions chosen to produce emission from K-shell resonance and satellite lines from He- to F-like Ti. All of the spectra with hot electrons have smaller bulk electron temperatures and emission from a wider range of charge states than the spectra without hot electrons. (H-like Ti emission, although 100 times more intense in the spectrum with hot electrons than in the single-temperature spectrum in Fig. 6 (a), is weak at the given conditions and is not shown.) In all three cases, the spectra with hot electrons have larger absolute intensities than the spectra without hot electrons but have been normalized to illustrate the effects of hot electrons on relative line intensities.

Satellite lines can be formed either by direct inner-shell excitation from the ground or low-lying levels of the ion with charge $z$ or by dielectronic recombination from the ion with charge $z+1$. Lower-energy satellite lines are often formed primarily by the latter process because their lower levels (the sources of the strongest collisional excitation rates) lie above the ground state of the parent ion and are less populated than the ground states, especially at lower electron densities. For instance, the Li-like satellites labeled $j$ and $k$ in Fig. 6 (a) are transitions from $1 s 2 p^{2}$ to $1 s^{2} 2 p$ and are primarily formed by dielectronic recombination from the He-like ground state, while the higher-energy satellites labeled $q$ and $r$ are $1 s 2 s 2 p-1 s^{2} 2 s$ transitions formed primarily by inner-shell excitation. In all of the spectra shown in Fig. 6, the inner-shell (high-energy) satellites are amplified in the spectra with hot electrons relative to the same satellites in the spectra without hot electrons. These effects, which have been noted for K-shell spectra of Ti, Ar, 
and $\mathrm{Mg},{ }^{18} 2123$ are not an unambiguous signature of hot electrons, since inner-shell satellite lines may also be intense in high-density, single-temperature plasmas. ${ }^{23}$

Finally, Fig. 6 (a) shows that hot electrons can have a marked effect on the intensity of the He-like intercombination line ( $\left.\mathrm{He}_{\mathrm{IC}}\right)$. At low electron densities, $\mathrm{He}_{\mathrm{IC}}$ is fed by radiative cascades from highly excited He-like levels that cannot decay directly to the ground state. The ratio of $\mathrm{He}_{\mathrm{IC}}$ to the resonance line $\mathrm{He}_{\alpha}$ is often used as a density diagnostic, since as the electron density increases, increased collisions between excited levels disturb the radiative cascade process and the level populations approach statistical equilibrium, at which $\mathrm{He}_{\mathrm{IC}}$ is much less intense than $\mathrm{He}_{\alpha}$. Hot electrons increase the total excitation into excited levels and thereby increase the number of radiative cascades, leading to a higher $\mathrm{He}_{\mathrm{IC}} / \mathrm{He}_{\alpha}$ ratio than would be expected at a given plasma density without hot electrons. This effect on $\mathrm{He}_{\mathrm{IC}}$ was previously noted for He-like Mg. ${ }^{23}$ Because in the absence of hot electrons, intense intercombination lines imply low electron densities and intense inner-shell satellite lines imply high electron densities, the simultaneous presence of both features in experimental spectra are strong evidence for the presence of hot electrons.

The influence of hot electrons on L-shell spectra from $\mathrm{Kr}(4-2)$ and Mo (3-2) is shown in Fig. 7 (a) and (b), respectively. Figure 7 (a) shows that hot electrons amplify inner-shell (higher-energy) satellite lines from $\mathrm{Na}$ - and $\mathrm{Mg}$-like charge states as compared with the satellite structures typically seen from a single-temperature plasma, an effect similar to that observed in the K-shell Ti spectra of Fig. 6. In Fig. 7 (a), hot electrons also amplify 
the Ne-like lines that are fed partially by radiative cascades ( $4 \mathrm{~F}$ and $4 \mathrm{G}$ ) relative to the Ne-like lines that have intensities limited by direct collisional excitation (4C and 4D). Finally, the hot electrons increase F-like Kr emission without significantly decreasing emission from Mg-and Na-like Kr. Figure 7 (b) illustrates similar effects on 3-2 emission from Mo: hot electrons increase the intensities of the cascade-fed resonance lines (3A, $3 \mathrm{~B}, 3 \mathrm{~F}$, and $3 \mathrm{G}$ ) relative to the excitation-limited $3 \mathrm{C}$ and $3 \mathrm{D}$ lines and increase F-like emission while retaining significant emission from lower charge states. The Ne-like 3F and $3 \mathrm{G}$ lines are sensitive to the electron density in the same manner as the He-like intercombination line. However, because the $3 \mathrm{~A}$ and $3 \mathrm{~B}$ lines are also fed partially by cascades from higher levels, a density diagnostic based on the ratio $(3 \mathrm{~A}+3 \mathrm{~B}) /(3 \mathrm{~F}+3 \mathrm{G}){ }^{19}$ is not as sensitive to the presence of hot electrons as a diagnostic based on the ratio $\mathrm{He}_{\mathrm{IC}} /$ $\mathrm{He}_{\alpha}$.

The increased populations of excited levels due to excitation by hot electrons suggests a further diagnostic tool for their detection that may be especially significant in low-density plasmas. Transitions between closely spaced levels of different configurations can be measured in the same spectral range; the presently considered cases have such transitions in the UV/EUV range. The relative intensities of lines from such inter-configuration complexes could be used to infer the relative populations of the configurations and thereby to detect the presence of hot electrons. ${ }^{37}$ 


\section{EFFECTS OF EDF ON CONTINUUM AND INNER-SHELL LINE EMISSION}

The signatures of hot electrons on charge state balances and line emission spectra discussed above are due to the effects of hot electrons on collisional bound-bound (excitation) and bound-free (ionization) rates. Hot electrons also affect the free-free and free-bound processes that give rise to continuum radiation. Free-free bremsstrahlung radiation in particular can be used as an unambiguous indicator of the presence of hot electrons in plasma. In the figures below, the radiative recombination intensity is calculated from steady-state level populations and self-consistent radiative recombination cross sections. The bremsstrahlung emission intensity is determined using the formalism given in ${ }^{38}$ with free-free Gaunt factors approximated by a weighted average of the Kramers and Born values for Maxwellian distributions and the Born-Elwert formulation for non-Maxwellian distributions.

Figure 8 shows the effects of including different fractions of hot electrons on line and continuum emission calculated using an L-shell Mo model based on data from the atomic structure code FAC ${ }^{32}$ that includes detailed atomic structure for F-, Ne- and Na-like Mo including $17-20 \mathrm{keV} \mathrm{K}$-shell transitions formed by excitation of a 1s electron in Ne-like Mo (for more details, see ${ }^{31}$ ). The hot electrons are in a Maxwellian distribution with $\varepsilon_{0}=$ $30 \mathrm{keV}$ and the bulk electrons have $T_{e}=800 \mathrm{eV}$ and the electron density is $10^{21} \mathrm{~cm}^{-3}$. The spectrum without hot electrons, given in Fig. 8 (a), has continuum emission that decays very quickly at high photon energies and negligible K-shell line intensities. In Fig. 8 (b), 
a fraction of hot electrons too small to significantly impact the average ion charge $\left(f=10^{-}\right.$ ${ }^{5}$ ) is shown to considerably increase the intensities of the high-energy continuum and Kshell lines. A still larger fraction of hot electrons fractions $\left(f=10^{-3}\right)$ has a correspondingly larger effect on both high-energy continuum and K- shell emission intensities, as shown in Fig. 8 (c).

In Fig. 9, the high-energy behavior of the bremsstrahlung radiation resembles the highenergy behavior of the underlying Maxwellian electron energy distribution. This tendency carries over to the other distribution functions as well, as shown in Fig. 9, which gives modeled Mo line and continuum emission calculated using the three different distribution functions given in equations (1) - (3). The spectra in Fig. 9 were calculated using pure forms of the different distributions (i.e. $f=1$ ) with $\varepsilon_{0}\left(T_{e}\right)=1 \mathrm{keV}$ and $n_{e}=$ $10^{21} \mathrm{~cm}^{-3}$. In all three cases, the continuum emission reflects the characteristics of the electron distribution function. While bremsstrahlung emission from the broad $\left(w=\varepsilon_{0}=1\right.$ keV) Gaussian EDF shown in Fig. 9 (a) decays rapidly after $2 \mathrm{keV}$, the bremsstrahlung emission from the Maxwellian and power-law distributions shown in Fig. 9 (b) and (c) remain intense up to much larger photon energies. The power-law distribution, which has more high-energy electrons than either the Gaussian or Maxwellian distributions, is the only distribution that gives significant K-shell line radiation. Radiative recombination features in the three spectra also tend to mirror the functional form of the underlying electron energy distribution. 
The spectra shown in Fig. 9 were all calculated with distributions whose characteristic energy $(1 \mathrm{keV})$ is not larger than the transition energies of interest. The fact that the charge state balances in these cases vary significantly with the functional form of the electron distribution underscore that the form of the distribution is insignificant only if its characteristic energy is larger than the largest transition energy. In this case, the ionization potential of Na-like Mo $(1790 \mathrm{eV})$ is small enough that some electrons in the broad Gaussian distribution can directly ionize the Na-like Mo ions. However, ionization of Ne-like Mo $\left(E_{z}=4250 \mathrm{eV}\right)$ by electrons in the Gaussian distribution is practically prohibited, so the average ion charge of the modeled plasma with the Gaussian distribution is highly constrained. The broader distributions have sufficient numbers of high-energy electrons to push the average ion charge significantly higher, up to near Nelike Mo. The effect of the functional form of the electron energy distribution is greatest at low densities where ladder ionization is least important - an effect seen experimentally in emission spectra from electron beam ion traps (EBITs), which have electron densities near $10^{12} \mathrm{~cm}^{-3}$. Typically, EBITs excite electrons with practically monoenergetic beams that resemble narrow Gaussian distributions, however, EBIT devices can also simulate arbitrary electron distribution functions by sweeping the beam energy and find that for a given characteristic energy, broader distributions reach higher charge states. As an illustration, He-like Ti emission has been observed at the Livermore EBIT using either a monoenergetic beam at $4.8 \mathrm{keV}$ or a pseudo-Maxwellian distribution with a much smaller characteristic energy around $2.3 \mathrm{keV} .{ }^{39} 4041$ 
Unlike hot electron diagnostics based on charge state balances and x-ray line spectra, the continuum-based hot electron diagnostics discussed here are not limited by the bulk temperature required to reach the L- or K-shell of a given element. Therefore, measurements of high-energy emission can be used to detect arbitrarily small fractions of hot electrons and are limited only by detector sensitivity and range. And while experimental continuum spectra cannot in general be inverted to determine a unique electron distribution function, they can be analyzed to infer properties of the electron distribution such as limiting values for energy cutoffs and the functional energy dependence of the distribution at high electron energies.

Measurements of line and continuum emission emission can be used to infer the presence of hot electrons and some properties of their energy distribution. An additional tool that can be used to characterize hot electrons in spatially anisotropic distributions is spectropolarimetry (see, for example, ${ }^{4041424344}$ ). If an electron beam is present in a plasma, line emission from particular transitions formed by collisional interaction with the beam electrons may be polarized preferentially along the beam axis. Line polarization is dependent on both the energy and spatial distribution of the impact electrons. Thus, by measuring parallel and perpendicular components of plasma emission simultaneously, the anisotropy of a hot electron distribution can be determined and limits can be imposed on the energies of the beam electrons. 


\section{SUMMARY AND CONCLUSIONS}

The effects of various electron distribution functions on plasma collisional rates, charge state balances, and x-ray emission have been investigated in the previous sections with an emphasis on isolating practical techniques to detect and characterize hot electrons in experimental plasma sources. In particular, plasmas with cool bulk Maxwellian distributions and a small fraction $f$ of hot electrons were considered. Estimates of the sensitivity of K- and L-shell spectra to hot electron fractions from elements with atomic numbers $Z$ from 10 to 50 were obtained and compared to reported results. Lower- $Z$ elements require less energy in the bulk electron distribution to ionize to their K- or Lshell and are thus better choices for experiments in which small fractions of hot electrons must be diagnosed from line spectra.

On the broad topics of charge state distributions and K- and L-shell spectral line emission as determined by collisional-radiative atomic kinetics models, several important effects of hot electrons were shown. Hot electrons increase and spread out the charge state balance of a plasma at a given bulk electron temperature. Emission lines and features from single charge states are also affected by hot electrons and are particularly useful in distinguishing the presence of hot electrons from other plasma phenomena such as spatial and temporal gradients and opacity effects. The effects of hot electrons on line spectra include amplification of the intensities of high-energy lines (including inner-shell emission lines), inner-shell satellite lines, and lines fed by radiative cascades in closedshell ions. 
The effects of varying the functional form and characteristic energy of the hot electron distribution were investigated. First, it was shown that most collisional rates are much more sensitive to the fraction of hot electrons $f$ than to the exact functional form or characteristic energy $\varepsilon_{0}$ of the hot electrons as long as $\varepsilon_{0}$ is larger than the largest relevant transition energy. The results of collisional-radiative models are also more sensitive to $f$ than to the other characteristics of the hot electron distribution. Second, the effects of a given fraction of hot electrons on modeled charge state distributions and spectral line emission decrease with increasing bulk electron temperatures and densities. Larger bulk temperatures naturally include larger numbers of energetic electrons and therefore require larger fractions of hot electrons to show significant effects. Larger electron densities move level populations towards Boltzmann equilibrium, where collisions among excited levels can have more impact on the population dynamics than high-energy collision processes and radiative decays facilitated by hot electrons, thereby decreasing the impact of hot electrons.

Line spectra from collisional-radiative models can be used to detect the presence and approximate number of hot electrons in experimental plasmas. To determine more precisely the characteristics of the hot electron distribution, high-energy continuum and spectropolarimetry can be used. High-energy bremsstrahlung emission can help to determine the number of hot electrons and the general shape, characteristic energy, and energy cutoff of the distribution. Spectropolarimetry can be used to diagnose characteristic energies and anisotropy in hot electron distributions. With sufficiently 
sophisticated measurements and modeling, the electron distributions in plasma sources can be well characterized.

\section{ACKNOWLEDGEMENTS}

The authors thank Kevin Fournier for helpful discussions and comments on this work. This work was supported by Lawrence Livermore National Laboratory under contract B520743 and by the DOE Cooperative Agreement DE-FC08-01NV14050. The work of S. H. was performed in part under the auspices of the U.S. Department of Energy by University of California Lawrence Livermore National Laboratory under contract No. W7405-Eng-48.

\section{APPENDIX. THREE-BODY RECOMBINATION}

Three-body recombination, the reverse process of collisional ionization, is an important process in high-density plasmas. Two incident electrons participate in the recombination process: one that recombines with the ion and one that carries off the excess energy. For single-temperature Maxwellian distributions, both electrons come from the same

distribution and the three-body recombination rate coefficients $\left\langle\left\langle\mathrm{v}_{1} \mathrm{v}_{2} \sigma^{3 b r}\right\rangle\right\rangle$ can be obtained from detailed balance of the collisional ionization rate: 


$$
g_{Z}\left\langle\mathrm{v} \sigma^{c i}\right\rangle=2\left(\frac{m_{e} T_{e}}{2 \pi \hbar^{2}}\right)^{3 / 2} g_{Z+1}\left\langle\left\langle\mathrm{v}_{1} \mathrm{v}_{2} \sigma^{3 b r}\right\rangle\right\rangle \exp \left[-E_{Z} / T_{e}\right]
$$

For multi-component electron distributions as discussed in the body of this paper, the two electrons that participate in three-body recombination may come from either the bulk Maxwellian distribution or the hot electron distribution. The calculation of the three-body recombination rate requires integrating a three-body recombination cross section $\sigma^{3 b r}$ over a multi-component electron distribution $F(\varepsilon)$ as given in (4):

$$
\left\langle\left\langle\mathrm{v}_{1} \mathrm{v}_{2} \sigma^{3 b r}\right\rangle\right\rangle=\int_{0}^{\infty} \int_{0}^{\infty} \mathrm{v}_{1} \mathrm{v}_{2} \sigma^{3 b r}\left(\varepsilon_{1}, \varepsilon_{2}\right) F\left(\varepsilon_{1}\right) F\left(\varepsilon_{2}\right) d \varepsilon_{1} d \varepsilon_{2} \quad\left[\mathrm{~cm}^{6} \mathrm{~s}^{-1}\right]
$$

In (A.2), the two electrons are distinguished with subscripts and $v$ is the incident electron velocity.

We are not aware of any simple analogue to the Klein-Rosseland formula (6) relating the doubly differential three-body recombination cross section $\sigma^{3 b r}$ to the collisional ionization cross section $\sigma^{c i}$. We here derive a simple expression for $\sigma^{3 b r}$ which satisfies detailed balance (A.1) and which can be used to obtain a three-body recombination rate coefficient for an arbitrary electron distribution function. After deriving the cross section, the dependence of three-body recombination rate coefficients on the functional form of electron distribution functions and their characteristic energies is investigated and analytical approximations are given for the double integral in (A.2). 
The three-body recombination cross section is derived by enforcing detailed balance (A.1) using the general expression for the double integration (A.2) with a Maxwellian distribution:

$$
\left\langle\left\langle v_{1} v_{2} \sigma^{3 b r}\right\rangle\right\rangle=\frac{8}{m_{e} \pi T_{e}^{3}} \int_{0}^{\infty} \int_{0}^{\infty} \varepsilon_{1} \varepsilon_{2} \sigma^{3 b r}\left(\varepsilon_{1}, \varepsilon_{2}\right) \exp \left[-\left(\varepsilon_{1}+\varepsilon_{2}\right) / T_{e}\right] d \varepsilon_{1} d \varepsilon_{2}
$$

Let $u=\varepsilon_{1}+\varepsilon_{2}, \varepsilon_{1}=\alpha u \equiv \mathrm{f}(\alpha, u)$, and $\varepsilon_{2}=(1-\alpha) u \equiv \mathrm{g}(\alpha, u)$. Then the double integral over $\varepsilon_{1}$ and $\varepsilon_{2}$ can be expressed as:

$$
\begin{gathered}
\left\langle\left\langle\mathrm{v}_{1} \mathrm{v}_{2} \sigma^{3 b r}\right\rangle\right\rangle=\frac{8}{m_{e} \pi T_{e}^{3}} \int_{0}^{\infty} \int_{0}^{1} \alpha(1-\alpha) u^{2} \sigma^{3 b r}\left(\varepsilon_{1}, \varepsilon_{2}\right) \exp \left[-(u) / T_{e}\right] J d \alpha d u \\
\text { where } J=\left|\frac{\partial \mathrm{f}}{\partial u} \frac{\partial \mathrm{g}}{\partial \alpha}-\frac{\partial \mathrm{f}}{\partial \alpha} \frac{\partial \mathrm{g}}{\partial u}\right|=u .
\end{gathered}
$$

If we assume that the three-body recombination cross section is dependent only on the sum of the energies of the incident electrons, then the integral over $\alpha$ in (A.4) can be evaluated, and the three-body recombination rate coefficient becomes:

$$
\left\langle\left\langle\mathrm{v}_{1} \mathrm{v}_{2} \sigma^{3 b r}\right\rangle\right\rangle=\left(\frac{1}{6}\right)\left(\frac{8}{m_{e} \pi T_{e}^{3}}\right) \int_{0}^{\infty} u^{3} \sigma^{3 b r}(u) \exp \left[-(u) / T_{e}\right] d u
$$

In order for this formulation to satisfy detailed balance (A.1), the three-body recombination cross section must be:

$$
\sigma^{3 b r}\left(\varepsilon_{1}+\varepsilon_{2}\right)=3 \frac{g_{Z}}{g_{Z+1}} \frac{\pi^{2} \hbar^{3}}{m_{e}} \frac{\left(\varepsilon_{1}+\varepsilon_{2}+E_{Z}\right)}{\left(\varepsilon_{1}+\varepsilon_{2}\right)^{3}} \sigma^{c i}\left(\varepsilon_{1}+\varepsilon_{2}+E_{Z}\right)\left[\mathrm{cm}^{4} \mathrm{~s}\right]
$$

Equation (A.6) can be used to obtain three-body recombination cross sections from arbitrary expressions for the collisional ionization cross section $\sigma^{c i}$. For example, the Lotz formula gives the functional dependence of $\sigma^{c i}$ on the incident electron energy $\varepsilon$ as 
$\ln [\varepsilon] / \varepsilon$. The associated three-body recombination cross section would depend on the sum of the incident electron energies as $\ln \left[\left(\varepsilon_{1}+\varepsilon_{2}+E_{\mathrm{Z}}\right)\right] /\left(\varepsilon_{1}+\varepsilon_{2}\right)^{3}$.

The rate of three-body recombination is given by $n_{e}{ }^{2}\left\langle\left\langle\mathrm{v}_{1} \mathrm{v}_{2} \sigma^{3 b r}\right\rangle\right\rangle$ for a single-component electron distribution. For the distribution specified in equation (4), composed of a Maxwellian distribution at temperature $T_{e}$ and a fraction $f$ of hot electrons in either a second Maxwellian, a Gaussian, or a power-law distribution with characteristic energy $\varepsilon_{0}$, the three-body recombination rate is:

$$
n_{e}^{2}\left[(1-f)^{2}\left\langle\left\langle\mathrm{v}_{1}^{M} \mathrm{v}_{2}^{M} \sigma^{3 b r}\right\rangle\right\rangle+2 f(1-f)\left\langle\left\langle\mathrm{v}_{1}^{M} \mathrm{v}_{2}^{X} \sigma^{3 b r}\right\rangle\right\rangle+f^{2}\left\langle\left\langle\mathrm{v}_{1}^{X} \mathrm{v}_{2}^{X} \sigma^{3 b r}\right\rangle\right\rangle\right]
$$

In (A.7), the superscripts on the incident electron velocities indicate the distribution of the numbered electron. For example, in the first term, $\left\langle\left\langle\mathrm{v}_{1}^{M} \mathrm{v}_{2}^{M} \sigma^{3 b r}\right\rangle\right\rangle$, both incident electrons come from the bulk Maxwellian distribution. That term can be calculated using detailed balance (A.1). The second term, $\left\langle\left\langle\mathrm{v}_{1}^{M} \mathrm{v}_{2}^{X} \sigma^{3 b r}\right\rangle\right\rangle$, is a cross term with one electron from the bulk Maxwellian distribution and one electron from the hot electron distribution and must be determined by integrating equation (A.2) over two different electron distributions. In the last term, $\left\langle\left\langle\mathrm{v}_{1}^{X} \mathrm{v}_{2}^{X} \sigma^{3 b r}\right\rangle\right\rangle$, both electrons come from the hot electron distribution.

Because three-body recombination cross sections decay with increasing electron energy, the recombination terms that include one or both electrons from the hot electron distribution are smaller than the term with both electrons from the bulk Maxwellian distribution. Therefore, the total three-body recombination rate coefficient lies between 
$(1-f)^{2}\left\langle\left\langle\mathrm{v}_{1}^{M} \mathrm{v}_{2}^{M} \sigma^{3 b r}\right\rangle\right\rangle$ and $\left\langle\left\langle\mathrm{v}_{1}^{M} \mathrm{v}_{2}^{M} \sigma^{3 b r}\right\rangle\right\rangle$ (as noted in ${ }^{33}$ ). If $f$ is small or $\varepsilon_{0}$ is much larger than $T_{e}$, minimal error is introduced by setting the total three-body recombination rate to $n_{e}^{2}(1-f)^{2}\left\langle\left\langle\mathrm{v}_{1}^{M} \mathrm{v}_{2}^{M} \sigma^{3 b r}\right\rangle\right\rangle$.

For fractions of hot electrons larger than a few percent and $\varepsilon_{0}$ less than about $100 T_{e}$, the trailing terms in equation (A.7) may make significant contributions to the total three-body recombination rate. Figure A.1 (a) shows the rate coefficient terms $\left\langle\left\langle\mathrm{v}_{1}^{X} \mathrm{v}_{2}^{X} \sigma^{3 b r}\right\rangle\right\rangle$ numerically integrated over various electron distributions as a function of the characteristic hot electron energy $\varepsilon_{0}$. The terms decay quickly with increasing $\varepsilon_{0}$ and are not particularly sensitive to the functional form of the electron distribution. The Maxwellian rate coefficients can be obtained using detailed balance with an electron temperature of $\varepsilon_{0}$. The Gaussian distributions give rate coefficients that are within $30 \%$ of the Maxwellian distribution for $\varepsilon_{0} / E_{\mathrm{Z}}>>1$ and can be approximated analytically by integrating over two delta functions at $\varepsilon_{0}$. For $\varepsilon_{0} / E_{\mathrm{Z}}>>1$, the delta function approximation agrees with numerical integration over the given Gaussian distributions to a few percent and to within $35 \%$ for the narrow power-law distribution with $\gamma=5$.

Three-body recombination rate coefficients for the cross terms $\left\langle\left\langle\mathrm{v}_{1}^{M} \mathrm{v}_{2}^{X} \sigma^{3 b r}\right\rangle\right\rangle$ with hot electrons at $\varepsilon_{0}$ and a bulk Maxwellian at $T_{e}=100 \mathrm{eV}$ are shown in Fig. A.1 (b). The cross terms with non-Maxwellian hot electrons can be analytically approximated by integrating over two delta functions at $T_{e}$ and $\varepsilon_{0}$. For $\varepsilon_{0} / E_{Z}>>1$, this approximation gives agreement with numerical integration to within $10 \%$ for the Gaussian distributions and $30 \%$ for the 
power-law distribution with $\gamma=5$. The bi-Maxwellian cross terms decrease less rapidly with $\varepsilon_{0}$ than the other terms because they include more low-energy electrons. They can be approximated by detailed balance (A.1) at an effective electron temperature $T_{e f f}^{4}=\left[\left(T_{e}+\varepsilon_{0}\right) / 2\right]^{3} \sqrt{T_{e} \varepsilon_{0}}$. This approximation agrees with numerical integration to better than $30 \%$ for ratios of $\varepsilon_{0} / T_{e}$ up to 10 . For larger ratios of $\varepsilon_{0} / T_{e}$, the effective temperature approximation for the bi-Maxwellian cross term is less reliable. However, because the cross term becomes negligible for large $\varepsilon_{0} / T_{e}$, the total recombination rate using this approximation will have errors of only a few percent in the worst case with $f=$ $50 \%$ and $\varepsilon_{0} / T_{e}>10$.

${ }^{1}$ R. Bartiromo, F. Bombarda, and R. Giannella, Phys. Rev. A 32, 531 (1985).

2 J. M. Liu, J. S. De Groot, J. P. Matte, T. W. Johnston, and R. P. Drake, Phys. of Plasmas 1, 3570, (1994).

${ }^{3}$ A. G. Zhidkov, A. Sasaki, T. Tajima, T. Auguste, P. D’Olivera, S. Hulin, P. Monot, A. Ya. Faenov, T. A. Pikuz, and I. Yu. Skobelev, Phys. Rev. E 60, 3273 (1999).

${ }^{4}$ A. G. Zhidkov, A. Sasaki, I. Fukumoto, T. Tajima, T. Auguste, P. D'Oliveira, S. Hulin, P. Monot, A. Ya. Faenov, T. A. Pikuz, and I. Yu. Skobelev, Phys. Plasmas 8, 3718 (2001).

${ }^{5}$ K.G. Whitney and P.E. Pulsifer, Phys. Rev. E 47, 1968 (1993).

${ }^{6}$ P. Choi, C. Deeney, and C.S. Wong, Physics Letters A 128, 80 (1988).

${ }^{7}$ V.L. Kantsyrev, D.A. Fedin, A.S. Shlyaptseva, S. Hansen, D. Chamberlain, and N. Ouart, Phys. of Plasmas 10, 2519 (2003). 
${ }^{8}$ N. R. Pereira and K.G. Whitney, Phys. Rev. A 38, 319, (1988).

9 J.M. McTiernan and V. Petrosian, Astrophys. J. 359, 541 (1990).

10 U. Feldman, Physica Scripta 65, 185 (2002).

${ }^{11}$ T. Kato, T. Fujiwara, and Y. Hanaoka, Astroph. J. 492, 822 (1998)

12 M.G. Baring, Mon. Not. R. Astron. Soc. 253, 388 (1991).

13 P. Beiersdorfer, S.R. Elliott, A. Osterheld, T. Stohlker, J. Autrey, G.V. Brown, A.J. Smith, and K. Widmann, Phys. Rev. A 53, 4000 (1996).

${ }^{14}$ W. L. Kruer, The Physics of Laser Plasma Interactions (Addison Wesley, 1988).

15 A. B. Langdon, Phys. Rev. Lett. 44, 575 (1980).

16 R. W. Harvey, V. S. Chan, S. C. Chiu, T. E. Evans, M. N. Rosenbluth, and D. G.

Whyte, Phys. of Plasmas 7, 4590 (2000).

17 J. Abdallah, Jr., A.Ya. Faenov, D. Hammer, S.A. Pikuz, G. Csanak, and R.E.H. Clark, Phys. Scr. 53, 705 (1996).

18 J. Abdallah, Jr., A.Ya. Faenov, I.Y. Skobelev, A.I. Magunov, T.A. Pikuz, T. Auguste, P. D’Oliveira, S. Hulin, and P. Monot, Phys. Rev. A 63, 032706 (2001).

19 A.S. Shlyaptseva, S.B. Hansen, V.L. Kantsyrev, D.A. Fedin, N. Ouart, K.B. Fournier, and U.I. Safronova, Phys. Rev. E 67, 026409 (2003).

20 A.H. Gabriel and K.J. Phillips, Mon. Not. R. Astron. Soc. 189, 319 (1979).

21 F.B. Rosmej, J. Phys. B 30, L819 (1997).

22 J. Abdallah, Jr., R.E.H. Clark, A.Ya. Faenov, L. Karpinski, S.A. Pikuz, V.M.

Romanova, M. Sadowski, M. Scholz, and A. Szydlowski, J. Quant. Spectrosc. Radiat.

Transf. 62, 85 (1999). 
23 J. Abdallah, Jr., A.Ya. Faenov, T.A. Pikuz, M.D. Wilke, G.A. Kyrala, and R.E.H. Clark, J. Quant. Spectrosc. Radiat. Transf. 62, 1 (1999).

${ }^{24}$ G. C. Junkel-Vives, J. Abdallah, Jr., T. Auguste, P. D’Oliveira, S. Hulin, P. Monot, S. Dobosz, A. Ya. Faenov, A. I. Magunov, T. A. Pikuz, I. Yu. Skobelev, A. S. Boldarev, and V. A. Gasilov, Phys. Rev. E 65, 036410 (2002).

${ }^{25}$ G.C. Junkel-Vives, J. Abdallah, Jr., F. Blasco, C. Stenz, F. Salin, A.Ya. Faenov, A.I. Magunov, T.A. Pikuz, I.Yu. Skobelev, T. Auguste, P. D’Oliveira, S. Hulin, P. Monot, and S. Dobosz, J.Quant. Spectrosc. Radiat. Transf. 71, 417 (2001).

${ }^{26}$ G.C. Junkel-Vives, J. Abdallah, Jr., F. Blasco, C. Stenz, F. Salin, A.Ya. Faenov, A.I. Magunov, T.A. Pikuz, and I.Yu. Skobelev, Phys. Rev. A 64, 021201 (2001).

27 S.H. Glenzer, F.B. Rosmej, R.W. Lee, C.A. Back, K.G. Estabrook, B.J. MacGowan, T.D. Shepard, and R.E. Turner, Phys.Rev. Lett. 81, 365 (1998).

28 S.B. Hansen, A.S. Shlyaptseva, A.Y. Faenov, I.Y. Skobelev, A.I. Magunov, T.A.

Pikuz, F. Blasco, F. Dorchies, C. Stenz, F. Salin, T. Auguste, S. Dobosz, P. Monot, P. D’ Oliveira, S. Hulin, U.I. Safronova, and K.B. Fournier, Phys. Rev. E 66, 046412 (2002).

${ }^{29}$ K.B. Fournier, A.Ya. Faenov, T.A. Pikuz, I.Yu. Skobelev, V.S. Belyaev, V.I. Vinogradov, A.S. Kyrilov, A.P. Matafonov, I. Bellucci, S. Martellucci, G. Petrocelli, T. Auguste, S. Hulin, P. Monot, and P. D'Oliveira, Phys. Rev. E . 67, 16402 (2003). ${ }^{30}$ K.B. Fournier, A.Ya. Faenov, T.A. Pikuz, I.Yu. Skobelev, F. Flora, S. Bollanti, P. Di Lazzaro, D. Murra, A. Reale, L. Reale, G. Tomassetti, A. Ritucci, I. Bellucci, S. Martellucci, G. Petrocelli, V.S. Belyaev, V.I. Vinogradov, A.S. Kyrilov, and A.P. Matafonov, JQSRT 81, 167 (2003). 
${ }^{31}$ S. B. Hansen, Ph.D. Dissertation, University of Nevada, Reno (2003).

${ }^{32}$ M. F. Gu, Astrophysical Journal 582, 1241 (2003).

${ }^{33}$ H.A. Kramers, Philos. Mag. 46, 836 (1923).

34 R. Epstein, S. Skupsky, and J. Delettrez, JQSRT 35, 131 (1986).

35 F.B. Rosmej, JQSRT 51, 319 (1994).

36 J.P. Matte, J.C. Kieffer, S. Ethier, M. Chaker, and O. Peyrusse, Phys. Rev. Lett. 72, 1208 (1994).

${ }^{37}$ U. Feldman, E. Landi, G. A. Doschek, I. Dammasch, and W. Curdt, Astrophys. J. 593, $1226(2003)$.

${ }^{38}$ U. Feldman, E. Landi, G. A. Doschek, I. Dammasch, and W. Curdt, Astrophys. J. 593, $1226(2003)$.

39 P. Beiersdorfer, G. Brown, S. Utter, P. Neill, K. J. Reed, A. J. Smith, and R. S. Thoe, Phys. Rev. A 60, 4156 (1999).

40 A.S. Shlyaptseva, V.L. Kantsyrev, B.S. Bauer, P. Neill, C.Harris, D.A. Fedin, S. Hansen, N. Ouart, P. Beiersdorfer, A.G. Petrashen, and U. I. Safronova, University of California Report No. UCRL-JC-146907, 339 (2001).

41 A. S. Shlyaptseva, D. A. Fedin, S. M. Hamasha, S. B. Hansen, C. Harris, V. L. Kantsyrev, P. Neill, N. Ouart, P. Beiersdorfer, and U. I. Safronova, Rev. Sci. Inst. 74, 1947 (2003).

42 J.C. Kieffer, J.P Matte, H. Pepin, M. Chaker, Y. Beaudoin, T.W. Johnston, C.Y. Chien, S. Coe, G. Mourou, and J. Dubau, Physical Review Letters 68, 480 (1992). 
${ }^{43}$ P. Hakel, R.C. Mancini, J.-C. Gauthier, E. Mi'nguez, J. Dubau, and M. Cornille, RSI 72 (2001).

${ }^{44}$ F. Dorchies, P. Forget, P. Gallant, Z. Jiang, J. C. Kieffer, H. Pe'pin, and O. Peyrusse, Phys. of Plasmas 8, (2001).

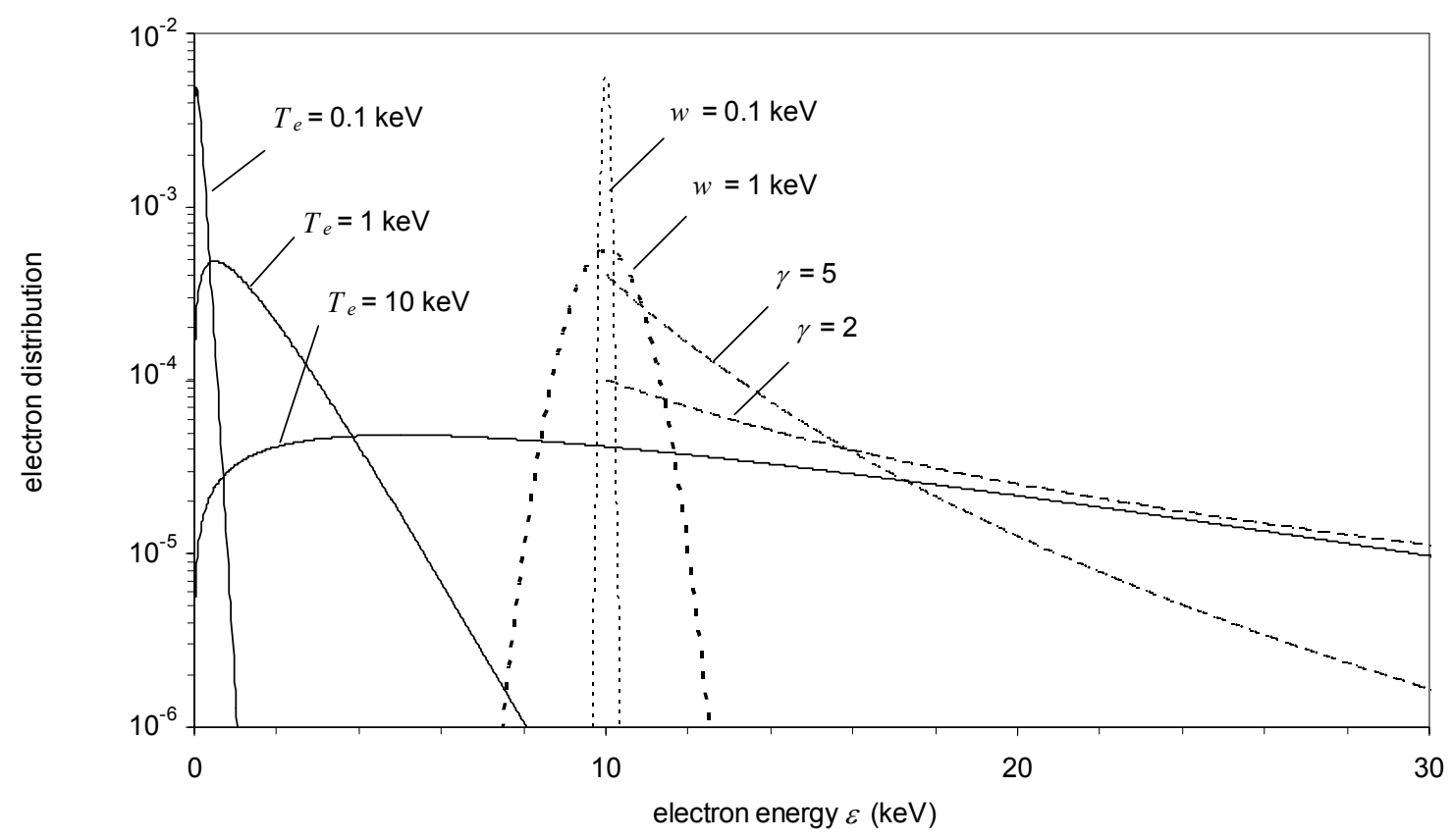

Fig. 1. Sample electron energy distribution functions: Maxwellian (solid lines) at various $T_{e}$, Gaussian (dotted lines) with $\varepsilon_{0}=10 \mathrm{keV}$ and various widths $w$, and power-law (dashed lines) with $\varepsilon_{0}=10 \mathrm{keV}$ and various decay constants $\gamma$. 

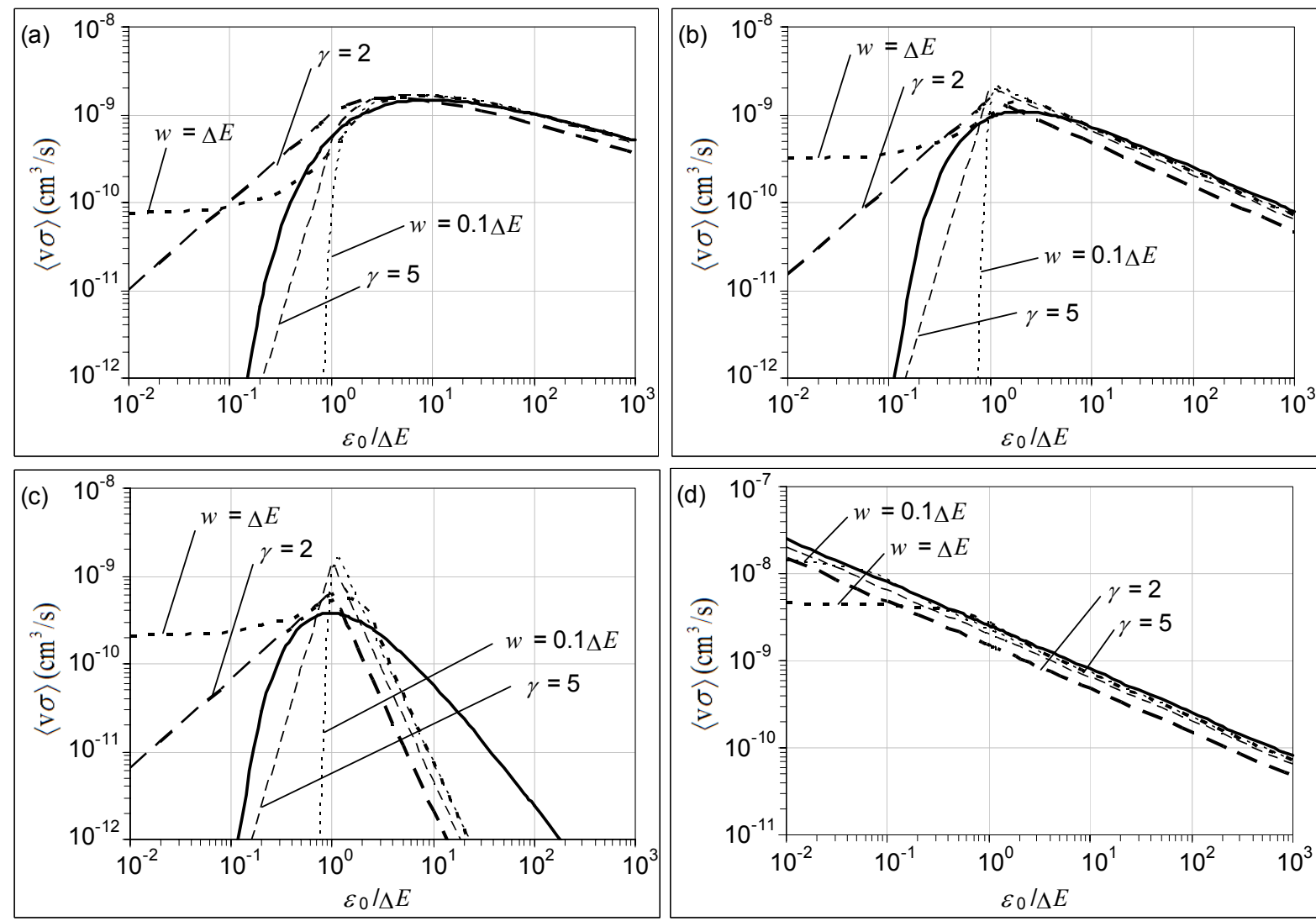

Fig. 2. Rate coefficients for various collisional processes: (a) resonant collisional excitation or collisional ionization, (b) - (c) non-resonant collisional excitation, and (d) collisional de-excitation or radiative recombination. The rate coefficients are obtained by integrating the given cross sections over Maxwellian (solid lines), Gaussian (dotted lines), and power-law (dashed lines) electron distribution functions and are given as a function of the characteristic distribution energy $\varepsilon_{0}$ in threshold units $\varepsilon_{0} / \Delta E$. In panels (a), (b), and (d), the value of $\varepsilon_{0}$ and the functional form of the electron distribution have a relatively minor influence on the collisional rates as long as $\varepsilon_{0} / \Delta E>1$. 

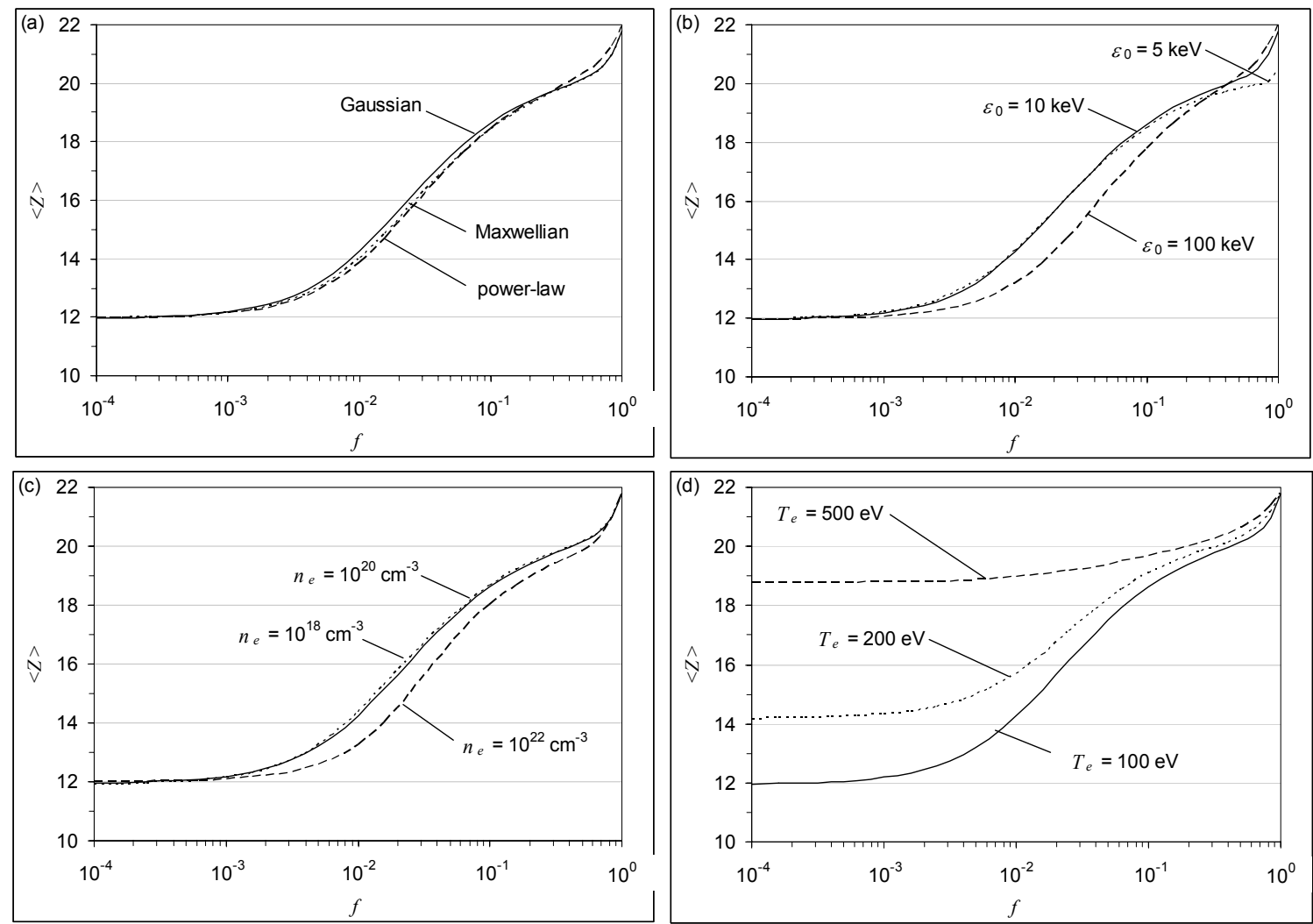

Fig. 3. The modeled average charge state $<Z>$ of Ti as a function of $f$ and the effects of various $T_{e}, n_{e}$, and hot electron distribution functions. The solid black lines in (a) - (d) have $T_{e}=100 \mathrm{eV}, n_{e}=10^{20} \mathrm{~cm}^{-3}$, and hot electrons in a Gaussian distribution with $\varepsilon_{0}=10 \mathrm{keV}$ and $w=100 \mathrm{eV}$. Dashed and dotted lines show the effects of changing: (a) the functional form of the hot electron distribution, (b) $\varepsilon_{0}$, (c) $n_{e}$, and (d) $T_{e}$ (as labeled). 


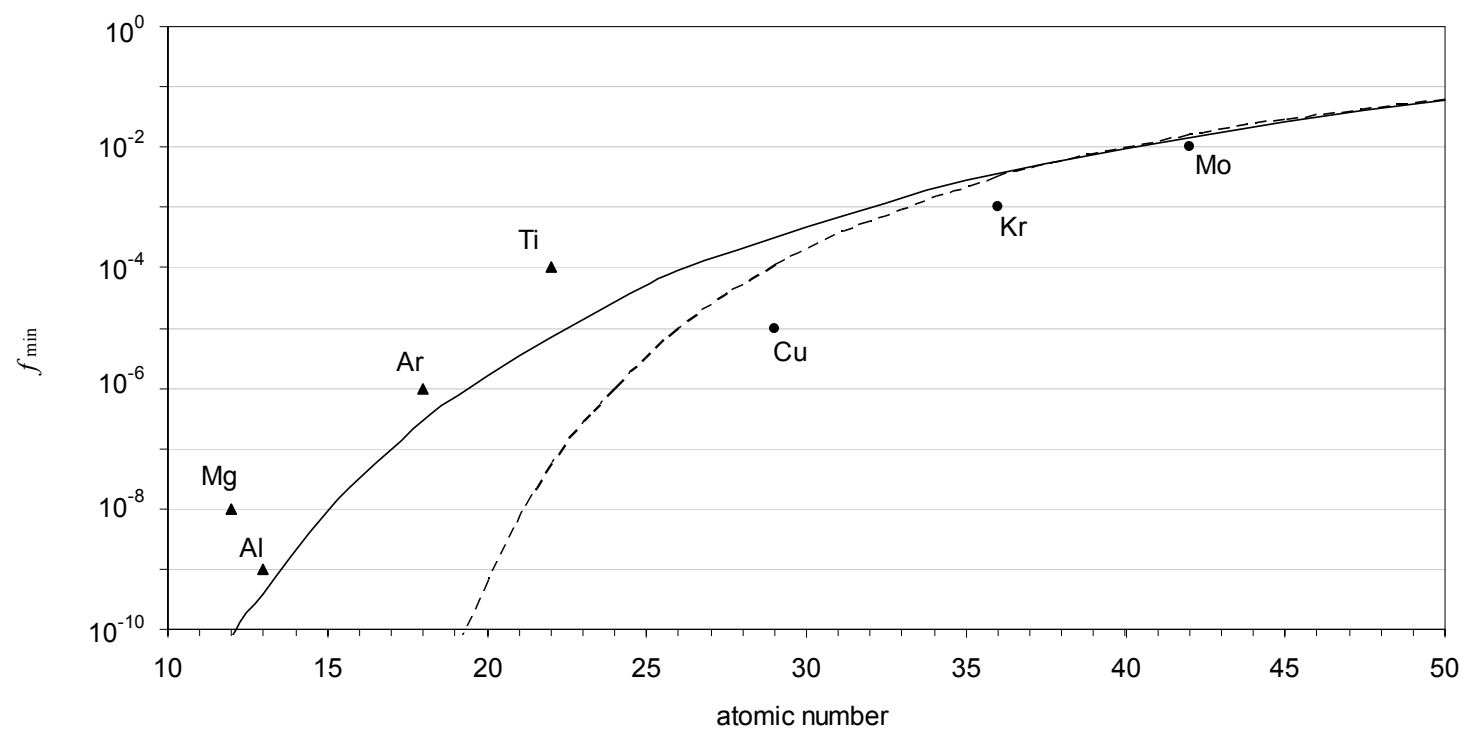

Fig. 4. Predicted values of the minimum hot electron fraction $\left(f_{\min }\right)$ to which modeled K-shell (solid line) and L-shell (dashed line) spectra are sensitive. Reported sensitivity limits of various collisional-radiative models are given by triangles for $\mathrm{K}$ shell Al, ${ }^{17} \mathrm{Mg},{ }^{23} \mathrm{Ar},{ }^{18}$ and $\mathrm{Ti}$ and by circles for $\mathrm{L}$-shell $\mathrm{Cu},{ }^{29} \mathrm{Kr},{ }^{28}$ and $\mathrm{Mo} .{ }^{19}$ 


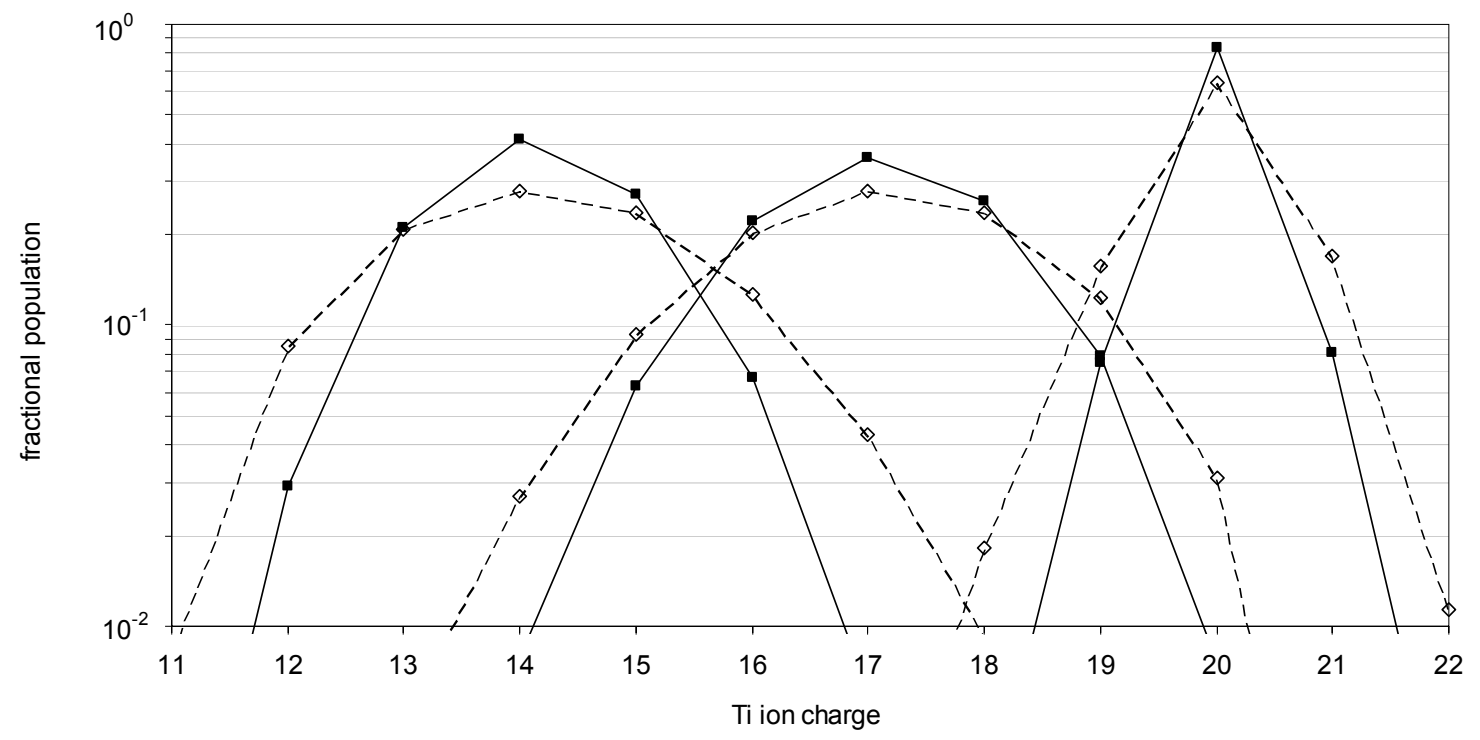

Fig. 5. Fractional populations of Ti ions modeled with $n_{e}=10^{20} \mathrm{~cm}^{-3}$ and EDF selected to obtain average charge states $<Z>$ near 14,17 , and 20 . Solid lines (closed squares) have $T_{e}=190,340$, and $2200 \mathrm{eV}$ and $f=0$. Dashed lines (open diamonds) have $T_{e}=100 \mathrm{eV}$ and $f=1 \%, 5 \%$, and $65 \%$ in a Gaussian distribution with $\varepsilon_{0}=10$ $\operatorname{keV}$ and $w=100 \mathrm{eV}$ 


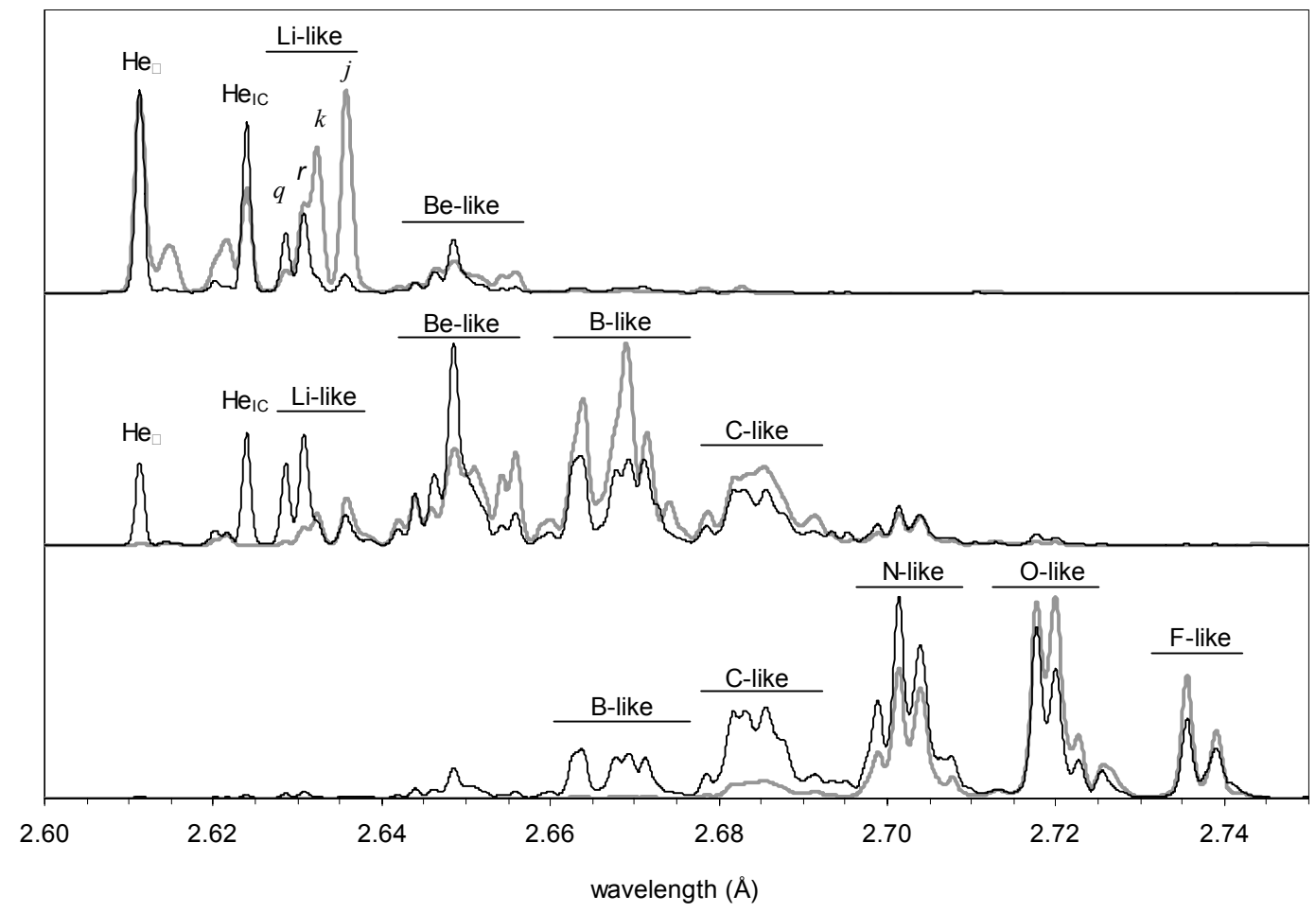

Fig. 6. Modeled Ti K-shell spectra with $n_{e}=10^{20} \mathrm{~cm}^{-3}$ and various electron distributions. Gray lines: $f=0$ and $T_{e}=$ (a) 800, (b) 350, and (c) $200 \mathrm{eV}$. Black lines: $T_{e}=100 \mathrm{eV}$ and hot electrons in a Gaussian distribution with $\varepsilon_{0}=10 \mathrm{keV}, w=100$ $\mathrm{eV}$, and $f=$ (a) $10 \%$, (b) $3 \%$, and (c) $1 \%$. 

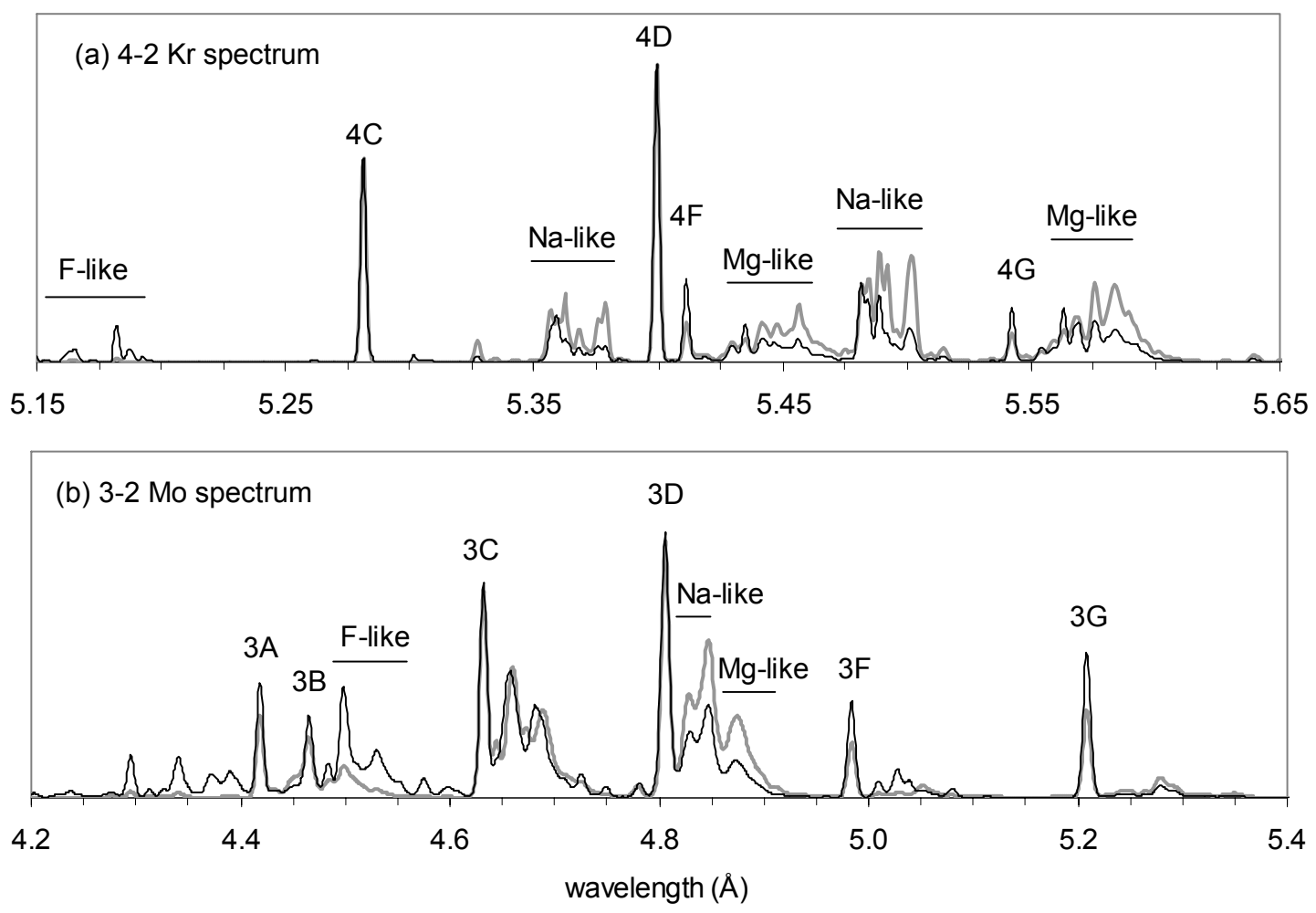

Fig. 7. Influence of hot electrons on L-shell spectra: (a) modeled Kr 4-2 spectra at $\boldsymbol{n}_{e}$ $=10^{20} \mathrm{~cm}^{-3}$ with $T_{e}=550 \mathrm{eV}$ and no hot electrons (gray lines) and $T_{e}=250 \mathrm{eV}$ and $5 \%$ hot electrons (black lines). The hot electrons are in a Gaussian distribution with $\varepsilon_{0}=20 \mathrm{keV}$ and $w=100 \mathrm{eV}$. (b) modeled Mo 3-2 spectra at $n_{e}=10^{22} \mathrm{~cm}^{-3}$ with $T_{e}=1$ keV and no hot electrons (gray lines) and $T_{e}=600 \mathrm{eV}$ and $10 \%$ hot electrons. The hot electrons are in a Gaussian distribution with $\varepsilon_{0}=30 \mathrm{keV}$ and $w=100 \mathrm{eV}$. 


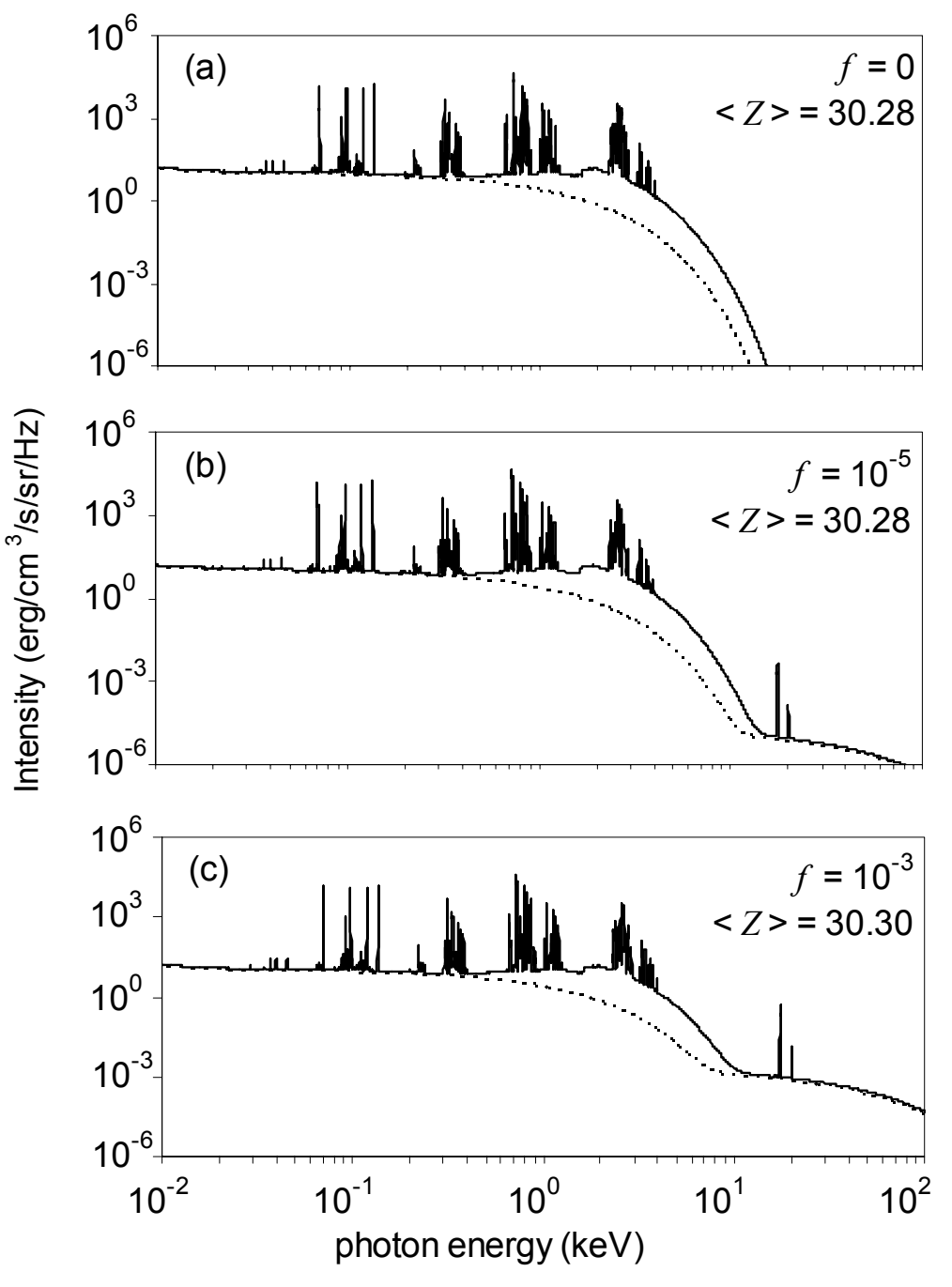

Fig. 8. Modeled Mo spectra calculated with a bulk Maxwellian at $T_{e}=800 \mathrm{eV}$ and the labeled fractions $f$ of hot electrons in a $30 \mathrm{keV}$ Maxwellian distribution $\left(n_{e}=10^{21}\right.$ $\left.\mathrm{cm}^{-3}\right)$. Even fractions of hot electrons too small to affect the L-shell line emission and average charge state can have significant influence of the intensities of inner-shell line and high-energy continuum emission. 


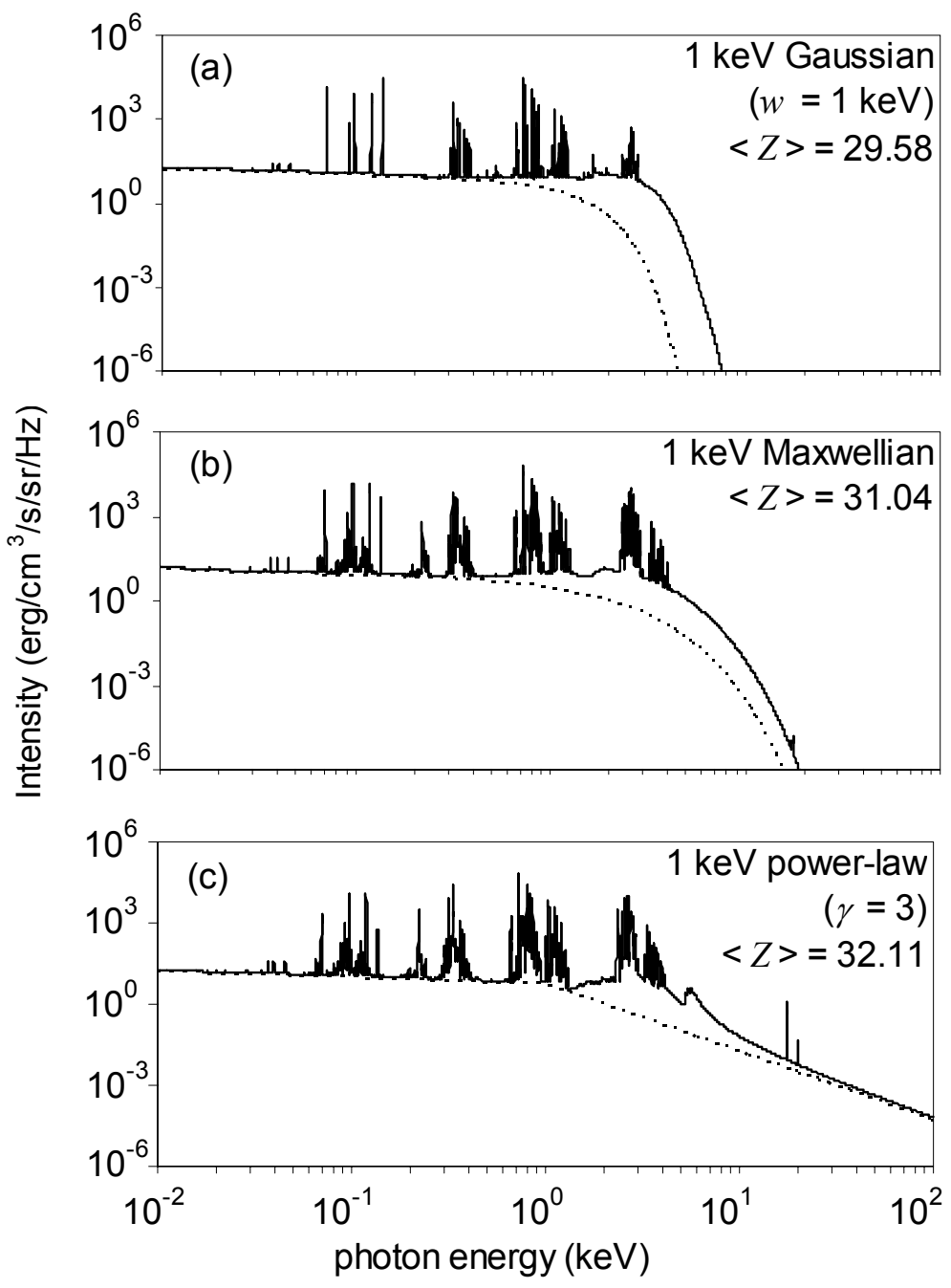

Fig. 9. Modeled Mo spectra calculated using different functional forms of the electron distribution (as labeled), all with $\varepsilon_{0}\left(T_{e}\right)=1 \mathrm{keV}$ and $n_{e}=10^{21} \mathrm{~cm}^{-3}$. Both the average ion charge and the high-energy bremsstrahlung emission are dependent on the functional form of the EDF. 

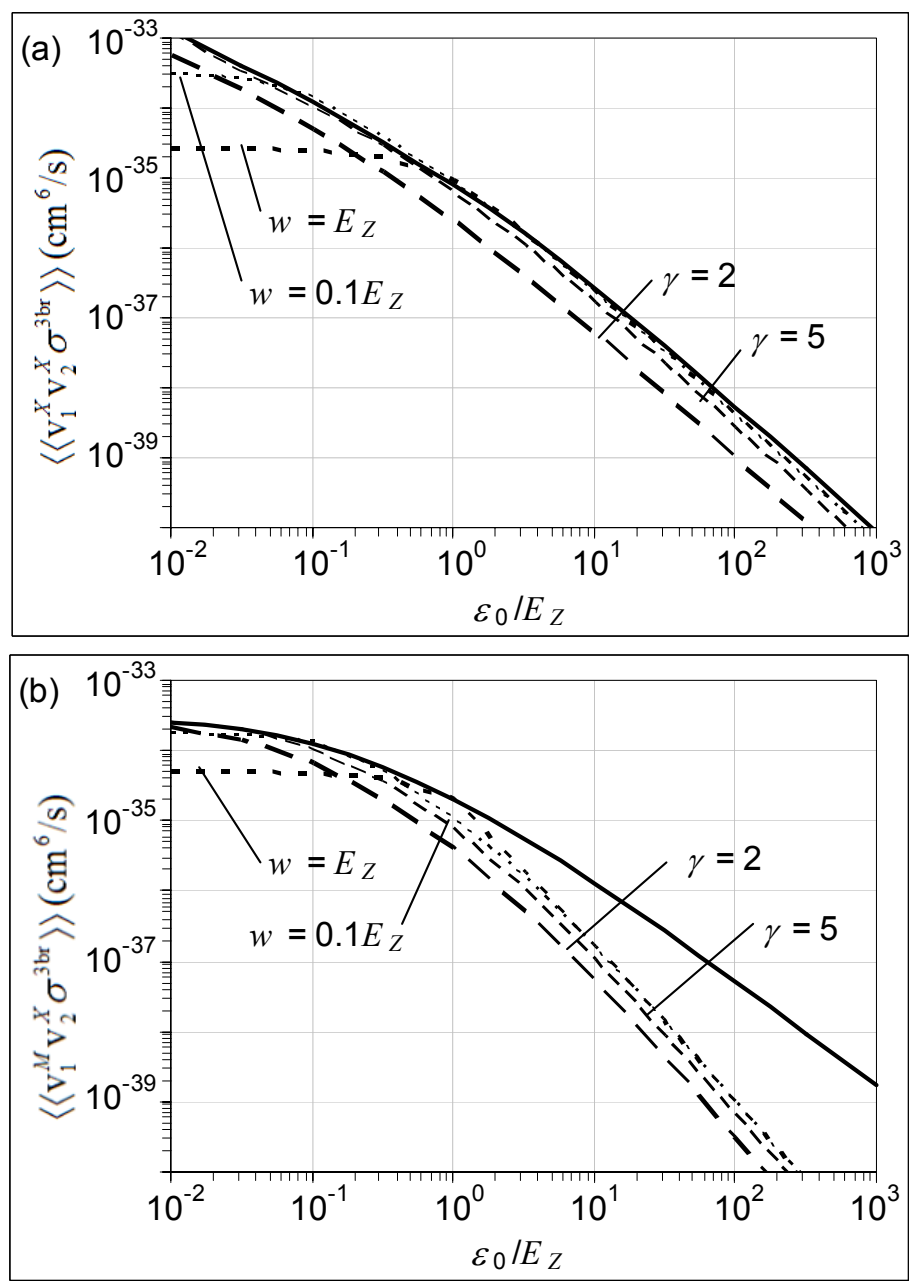

Fig. A.1. Three-body recombination rates for a transition with $E_{\mathrm{Z}}=1 \mathrm{keV}$ and ionization cross section $\sigma^{\mathrm{ci}}$ proportional to $\ln [\varepsilon] / \varepsilon$ : (a) Direct terms with both electrons from the same electron distribution. (b) Cross terms with one electron from a Maxwellian distribution with $T_{e}=100 \mathrm{eV}$ and the other from a different electron distribution. The rates are given as a function of the characteristic distribution energy $\varepsilon_{0}$ in threshold units for Maxwellian (solid lines), Gaussian (dotted lines), and power-law (dashed lines) electron distribution functions. 